\title{
Human-Nature Interaction in World Modeling with Modelica
}

\author{
Rodrigo Castro ${ }^{1,2}$, Peter Fritzson ${ }^{3}$, François Cellier ${ }^{4}$, Safa Motesharrei $^{5}$, Jorge Rivas ${ }^{6}$ \\ ${ }^{1}$ Department of Environmental Systems Science \\ ETH Zurich, Switzerland \\ ${ }^{2}$ Department of Computer Science, University of Buenos Aires \\ and CIFASIS-CONICET, Argentina \\ ${ }^{3}$ Department of Computer and Information Science \\ Linköping University, SE-581 83 Linköping, Sweden \\ ${ }^{4}$ Computer Science Department \\ ETH Zurich, Switzerland \\ ${ }^{5}$ National Socio-Environmental Synthesis Center (SESYNC) \\ Annapolis, MD, 21401, USA \\ ${ }^{6}$ Department of Political Science, University of Minnesota \\ Minneapolis, MN 55408, USA
}

\begin{abstract}
rodrigo.castro@usys.ethz.ch, peter.fritzson@liu.se, fcellier@inf.ethz.ch, ssm@umd.edu, jorgerodrigorivas@,gmail.com
\end{abstract}

\begin{abstract}
It is our predicament that we live in a finite world, and yet we behave as if it were infinite. Steady exponential material growth with no limits on resource consumption and population is the dominant conceptual model used by today's decision makers. This is an approximation of reality that is no longer accurate and started to break down. The World3 model, originally developed in the 1970s, includes many rather detailed aspects of human society and its interaction with a resourcelimited planet. However, World3 is a rather complex model. Therefore it is valuable for pedagogical reasons to show how similar behavior can be also realized with models that are much simpler. This paper presents a series of world models, starting with very simple exponential growth and predator-prey systems, then investigates a minimal human-nature model, Handy, and ends with a brief account of the World 3 model. For the first time, a simple human-nature interaction model is made available in Modelica that distinguishes between dynamics of Elite and Commoner social groups. It is shown that Handy can reproduce rather complex behavior with a very simple model structure, as compared to that of world models like World3.
\end{abstract}

Keywords: Modelica, Simulation, Population Dynamics, World Models, Human-Nature Models.

\section{Introduction}

Dynamic modeling can be applied to the human population and its interaction with the earth system. Certain types of such models are called World models.

Perhaps the earliest study indicating that human population would eventually reach limits to growth is that presented by Richard Malthus in 1798 in his famous work An Essay on the Principle of Population [4]. A catastrophe in the form of population collapse was considered as a possible consequence of uncontrolled growth that would hit the limits of food production causing massive famine.

A series of simulation models were developed mainly during the 1970 's, with some later updates, aiming at understanding the complexity of the interactions between global societies and their physical environment, searching for the conditions that would lead to a collapse and possible measures that can avoid it. They formed the category of so-called World Models, typically of considerable generality and complexity, spanning several subsystems (demography, energy, economy, industry, agriculture, etc.) at varied levels of detail. 


\section{Growth without Limitations}

In this first example, we shall show the dynamics of human populations in an idealized ecological system without limitations. We assume that a population contains $P$ individuals. In general, the rate of change of the number of individuals is the difference between the population birth rate and its death rate:

$$
\dot{P}=\text { birthrate }- \text { deathrate }
$$

where $\dot{P}$ means $d P / d t$, the rate of change of the population stock. It is natural to assume that the birth and death rates are proportional to the size of the population:

$$
\begin{aligned}
\text { birthrate } & =g \cdot P \\
\text { deathrate } & =d \cdot P
\end{aligned}
$$

where $g$ is the growth (birth) factor and $d$ the decrease (death) factor for the population. These factors can be assumed to be constant if the same amount of food per individual is available independent of the size of the population, and if no sudden lethal events (disease, war) affect the population massively. These assumptions are of course valid only for a reasonably limited size of the population, since food supply is never infinite in a closed system and epidemics, pandemics, and wars sometimes break out. Putting (1) and (2) together gives:

$$
\dot{P}=(g-d) \cdot P
$$

Solutions to this equation yield an exponentially increasing population if $(g-d)>0$ or an exponentially decreasing one if $(g-d)<0$. We represent this equation in the model class PopulationGrowth below:

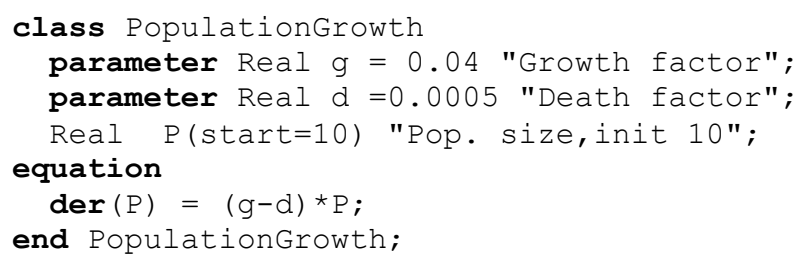

The model is simulated and the population size $P$ is plotted below:

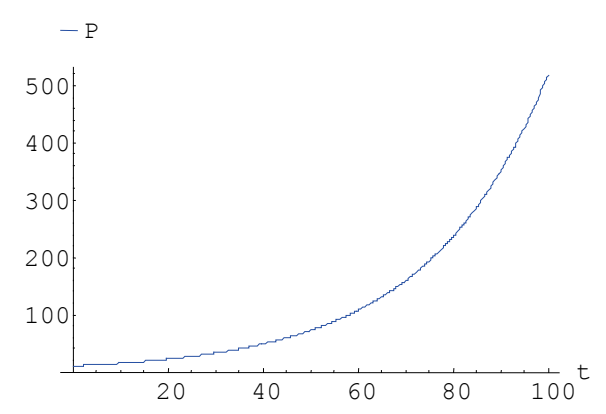

Figure 1. Exponential growth of a population with unlimited food supply.
As expected, Figure 1 shows an exponentially increasing population. We should, however, remember that exponential growth can never persist indefinitely in a closed system with a limited food supply.

\section{A Predator-Prey Model with Limi- tations in Prey Animal Population}

Now we will study a more interesting system consisting of interacting populations of predators and prey animals.

The simplest predator-prey model, the so-called Lotka-Volterra model [3], consists of two population stocks. We may think of predators as foxes and preys as rabbits.

The rabbit population of size $R$ is assumed to have an unlimited food supply. Therefore equation (3) applies regarding the birth rate of the rabbit population, with a positive growth term $g_{r} \cdot R$, where $g_{r}$ is the growth factor for rabbits.

On the other hand, the fox population of size $F$ feeds on the rabbits. The rabbit death rate can be assumed to be proportional to the number of foxes due to increased hunting pressure, and to the number of rabbits due to the higher probability of success in hunting, giving a rabbit death rate due to foxes of $d_{r f} \cdot F \cdot R$, where $d_{r f}$ is the death factor of rabbits due to foxes. Putting the birth and death rates together gives the total rabbit population change rate $\dot{R}$ as defined by equation (4) below:

$$
\dot{R}=g_{r} \cdot R-d_{r f} \cdot F \cdot R
$$

The growth of the fox population of size $F$ is proportional to the rate of rabbits consumed, i.e., those that die because they were hunted by foxes, which is the death rate term $d_{r f} \cdot F \cdot R$ mentioned in the previous equation. The efficiency of growing foxes from rabbits is determined by the growth factor $g_{f r}$, giving a fox population growth rate term $g_{f r} \cdot d_{r f} \cdot R \cdot F$. The fox population also has an intrinsic death rate $d_{f} \cdot F$ proportional to the size of the fox population by the fox death factor $d_{f}$. By combining these two terms, we obtain equation (5):

$$
\dot{F}=g_{f r} \cdot d_{r f} \cdot R \cdot F-d_{f} \cdot F
$$

where $\dot{F}$ is the total change rate of the fox population.

We have created a Modelica [2][8] class below called LotkaVolterra that includes both equations (4) and (5) along with all the variables and constant proportionality factors. The two state variables $R$ and $F$ for the sizes of rabbit and fox populations are represented by the Modelica variables rabbits and foxes: 


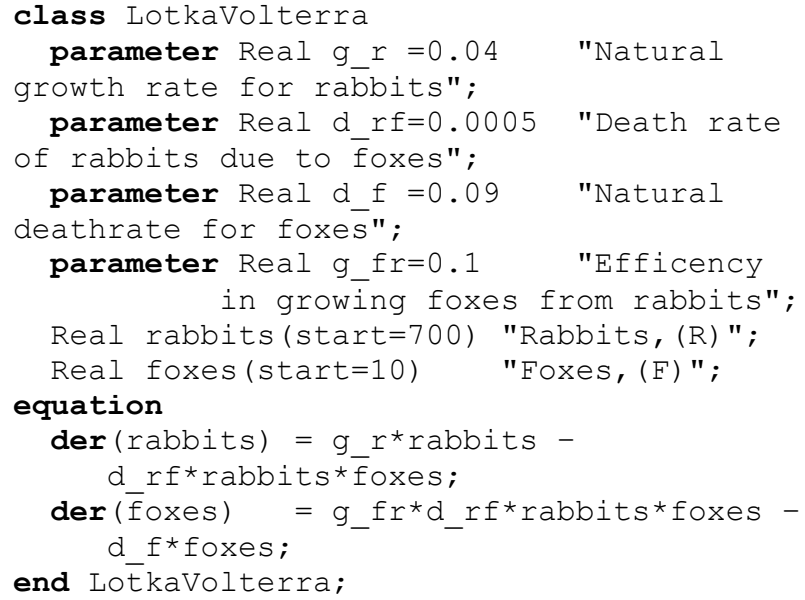

The model is simulated, and the sizes of the rabbit and fox populations as a function of time are shown below (Figure 2):

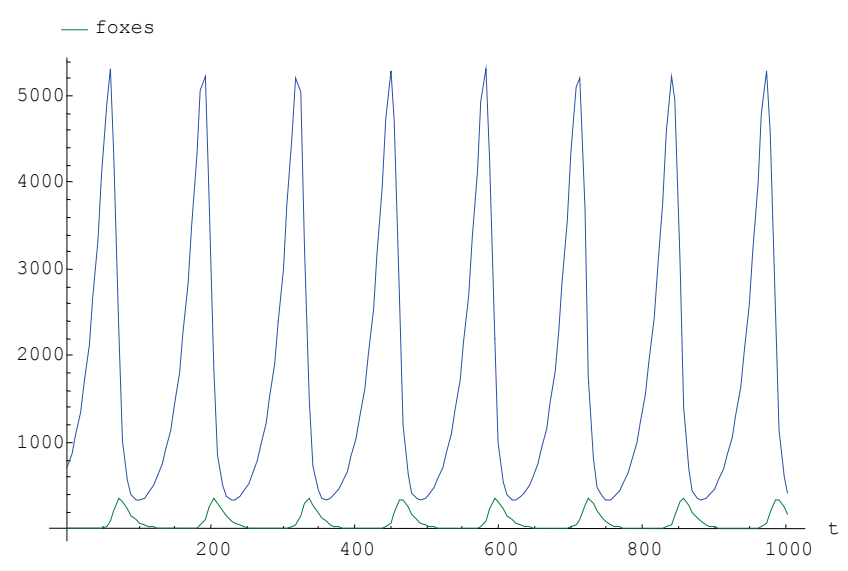

Figure 2. Number rabbits_ prey animals, and foxes - predators, as a function of time simulated from the PredatorPrey model.

The Lotka-Volterra model has a rather special property: the solution variables, i.e., the rabbit and fox population sizes, do not approach constant steady-state levels (unless they start at those equilibrium levels, which is rarely the case). Instead, they exhibit oscillatory equilibrium. The shape of these oscillations, very characteristic for Lotka-Volterra models, corresponds rather well to several experimental population studies of predators and prey animals. However, this does not prove these partly inductive model equations to be correct, only that they seem to have certain approximate physical significance.

\section{A Minimal Human-Nature Dynam- ical Model with Economic Stratifi- cation}

Simplified approaches to World models can exhibit interesting behavioral patterns while keeping model complexity low enough to enable intuitive comprehension. One such simplified model is Handy (Human and Nature Dynamical model) [9] developed jointly by researchers at the University of Maryland and the University of Minnesota ${ }^{1}$.

The LotkaVolterra model (Section 3) was the original inspiration behind Handy, which is not a world model in the typical sense, although, in principle, it can be applied to a homogenous world.

Handy has four differential equations describing the evolution of its state variables: Commoner population (commoners), Elite population (elite), regenerating natural resources (nature), and accumulated wealth (wealth). Human population plays a role analogous to that of predators, and nature plays the role of the resource preyed upon.

An interesting feature of Handy is that it introduces the accumulation of economic wealth, and divides the human population into rich and poor according to their unequal access to available wealth.

This new variable explains why human societies can undergo an irreversible collapse, while animal populations show cyclic changes (or reversible collapses).

Social inequality is not only explicitly considered but also plays a key role in the sustainability analyses of the model. This makes Handy the first model of its kind that studies the impacts of inequality on the fate of societies, a capability seldom found even in complex world models.

Handy establishes a useful general framework that allows carrying out "thought experiments" about societal collapse scenarios and the changes that might avoid them.

The model is a very strong simplification of the human-nature system, which results in many limitations. Despite its simplicity, such a model is easy to understand and offers a more intuitive grasp of underlying dynamical phenomena compared to more complex and less aggregated models.

We briefly introduce a much more advanced class of world model called World3 in Section 5 of this paper. This model (the compiled version) features 41 state variables and 245 algebraic variables. It captures many

\footnotetext{
${ }^{1}$ The Handy model has been publicly available since 2010 in the form of unpublished reports and presentations, deposited on the web site of its authors:

http://www.atmos.umd.edu/ ekalnay/\#publications based.
} 
aspects of the human system and ecology, and it has been calibrated against empirical data.

\subsection{Handy Model Equations, Description, and Modeled Societies}

The Handy model divides the total population into two classes: Commoners and Elites, of population sizes commoners and elite, respectively. Population grows through birth rates birthRComm and birthRElite, which are constants. The population shrinks through death rates deathRComm and deathRElite, which are modeled as functions of wealth.

The state variables of the model are described in Table 1, additional variables in Table 2 , and the parameters of the model in Table 3.

A single stock (variable), nature represents an amalgamation of nonrenewable and renewable resources, including "regenerating" resources such as forests, soils, and animal and fish stocks, etc.

The dynamics of the model are defined by the following four differential equations:

$$
\begin{gathered}
\text { commoners }{ }^{\prime}=\text { birthRComm } \cdot \text { commoners }- \text { deathRComm } \cdot \text { commoners } \\
\text { elite }{ }^{\prime}=\text { birthRElite } \cdot \text { elite }- \text { deathRElite } \cdot \text { elite } \\
\text { nature }{ }^{=} \text {natureRegen } \cdot \text { nature } \cdot(\text { natureCap }- \text { nature }) \\
\quad-\text { depletFactor } \cdot \text { commoners } \cdot \text { nature } \\
\text { wealth }{ }^{\prime}=\text { depletFactor } \cdot \text { commoners } \cdot \text { nature } \\
- \text { consRComm }- \text { consRElite }
\end{gathered}
$$

which in Modelica are expressed as:

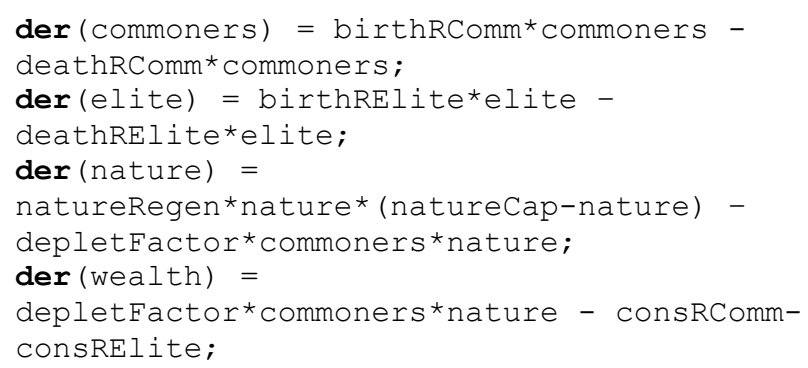

The equation for nature includes a regeneration term natureRegen*nature* (naturecap-nature) and a depletion term depletFactor*commoners*nature intended to also include degradation in nature caused by pollution.

The regeneration term is synthetic (i.e., not directly physical) following an s-shaped form ${ }^{2}$ parameterized to resemble physically realistic results.

It exhibits exponential growth for low values of nature (because natureCap-nature is almost constant for small nature), reaches its maximum at nature=natureCap/2, and becomes small when na-

2 This is the well-known "logistic equation" used in many domains of life sciences to represent exponential growth followed by smooth saturation. ture approaches naturecap (maximum size of nature in absence of depletion).

The depletion term includes a rate of depletion (pollution) per worker depletFactor making it proportional to both nature and commoners (labor). The economic activity of Elite is modeled to represent supervisory functions with no direct influence on the extraction of resources or the production of wealth. The underlying concept is that "only commoners produce." Handy does not model the impacts of technological change, considering that technology through history has proven to produce both increases and decreases in resource use efficiency. Thus, a simplification is made assuming that these effects cancel each other out.

Accumulated Wealth (wealth) increases with production (depletFactor* commoners*nature) and decreases according to the consumption rates of the Elites and the Commoners, consRComm and consRElite, respectively.

Table 1. Main state variables of the Handy model.

\begin{tabular}{|l|l|l|l|}
\hline Name & Description & Unit & $\begin{array}{l}\text { Typical ini- } \\
\text { tial value(s) }\end{array}$ \\
\hline commoners & $\begin{array}{l}\text { Commoner popula- } \\
\text { tion size }\end{array}$ & Number of people & 100 \\
\hline elite & Elite population size & Number of people & $0,1,25$ \\
\hline nature & Nature's stock & EcoDollars & $\begin{array}{l}\text { natureCap } \\
=100\end{array}$ \\
\hline wealth & Accumulated wealth & EcoDollars & 0 \\
\hline
\end{tabular}

The additional variables of the model are described in Table 2. Two of these variables describe consumption. The consumption of the Commoners, consRComm, is given by a subsistence salary per capita subssal times the working population. Elites earn a salary that is ineqFactor times larger than that of Commoners, intended to simulate class inequality, and is considered time-independent for any given scenario. This is reflected in the following equations:

consRComm $=\min (1$, wealth $/$ wealthMin $) \cdot$ subsSal $\cdot$ commoners consRElite $=\min (1$, wealth $/$ wealthMin $) \cdot$ subsSal $\cdot$ elite $\cdot$ ineqFactor

which in Modelica are expressed as:

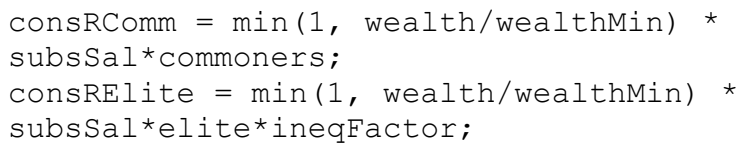

Both consumption rates, consRComm and consRElite, are subject to a "famine" minimum threshold for accumulated wealth before famine, wealthMin. The consumption rates are linearly reduced down to zero by the min () terms in the above equations, when wealth falls below wealthmin.

The death rates of the Commoners and the Elite are defined by the following equations: 
deathRComm $=$ deathRnormal $+\max (0,1-$ consRComm

$/($ subsSal $\cdot$ commoners $)) \cdot($ deathRfamine - deathRnormal $)$

deathRElite $=$ deathRnormal $+\max (0,1-$ consRElite

$/($ subsSal $\cdot$ elite $)) \cdot($ deathRfamine - deathRnormal $)$

The carrying capacity (carryingCap) of population, i.e., the population that can be sustained by the regrowth of nature, and its corresponding maximum carrying capacity (carryingCapMax), attainable under certain conditions, are defined by these equations:

carryingCap $=$ natureRegen $/$ depleteFactor .

(natureCap - subsSal $\cdot$ eta / depletFactor)

carryingCapMax $=$ natureRegen $/($ eta $\cdot$ subsSal $) \cdot(\text { natureCap } / 2)^{2}$

The threshold for minimum wealth (wealthMin), the total population, and a dimensionless quotient eta used above are defined as follows:

wealthMin $=$ consWorkerMin $\cdot$ commoners +

ineqFactor $\cdot$ consWorkerMin $\cdot$ elite

eta $=($ deathRfamine - birthRComm $) /$

(deathRfamine-deathRnormal)

totalPopulation $=$ elite + commoners

Table 2. Additional variables of the Handy model for Human and Nature Dynamics.

\begin{tabular}{|l|l|l|}
\hline Name & Description & Unit \\
\hline deathRComm & Commoners' death rate & $\begin{array}{l}\text { Number of peo- } \\
\text { ple/year }\end{array}$ \\
\hline deathRElite & Elites' death rate & $\begin{array}{l}\text { Number of peo- } \\
\text { ple/year }\end{array}$ \\
\hline consRComm & $\begin{array}{l}\text { Consumption rate of com- } \\
\text { moners }\end{array}$ & EcoDollars/year \\
\hline consRElite & Consumption rate of Elites & EcoDollars/year \\
\hline eta & Dimensionless quotient & $\begin{array}{l}\text { Number of people } \\
\text { whole system) }\end{array}$ \\
\hline carryingCap & Maximum carrying capacity & Number of people \\
\hline carryingCapMax & Number of people \\
\hline totalpopulation & Total population & \\
\hline
\end{tabular}

The parameters of the model are described in Table 3.

Table 3. Parameters of the Handy model for Human and Nature Dynamics.

\begin{tabular}{|l|l|l|}
\hline Name & Description & Typical value(s) \\
\hline deathRnormal & $\begin{array}{l}\text { Healthy normal (minimum) } \\
\text { death rate }\end{array}$ & 0.01 \\
\hline deathRfamine & Famine (maximum) death rate & 0.07 \\
\hline birthRComm & Commoner birth rate & 0.03 , or 0.065 \\
\hline birthRElite & Elite birth rate & 0.03 , or 0.02 \\
\hline subsSal & Subsistence salary per person & 0.0005 \\
\hline consWorkerMin & $\begin{array}{l}\text { Minimum required consump- } \\
\text { tion per worker }\end{array}$ & 0.005 \\
\hline natureRegen & Nature's regeneration rate & 0.01 \\
\hline
\end{tabular}

\begin{tabular}{|l|l|l|}
\hline natureCap & Nature's carrying capacity & 100 \\
\hline ineqFactor & $\begin{array}{l}\text { Inequality factor between elite } \\
\text { and commoners }\end{array}$ & 1,10, or 100 \\
\hline \multirow{2}{*}{ depletFactor } & Depletion (production) factor & $\begin{array}{l}6.67 \mathrm{E}-6, \text { or } \\
6.35 \mathrm{E}-6, \text { or } \\
13 \mathrm{E}-6\end{array}$ \\
\hline
\end{tabular}

There are three dimensions for quantities in the model: Population (either Commoners or Elites), in units of people, Nature or Wealth, in units of EcoDollars, and Time, in units of years. All other parameters and functions in the model carry units that are compatible with these dimensions.

All of the above definitions appear in the HandyBase Modelica model below, which is a partial model that is inherited by the following actual simulated models.

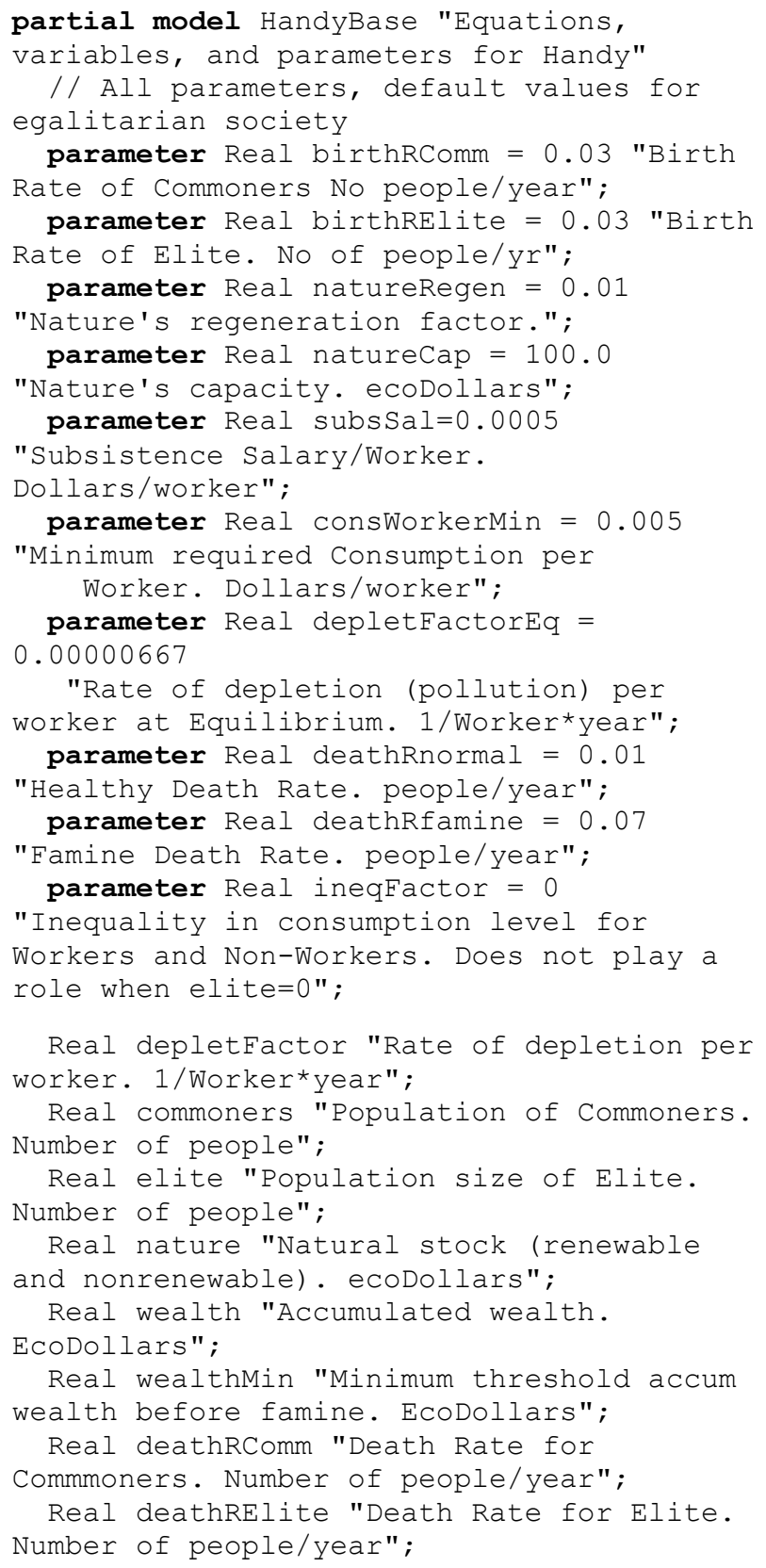


Real consRComm "Consumption Rate of Commoners. Dollars/year";

Real consRElite "Consumption Rate of

Elite. Dollars/year";

Real eta "Derived quotient expression.

Dimensionless";

Real carryingCap "Carrying Capacity.

Number of people";

Real carryingCapMax "Maximum Carrying

Capacity. Number of people";

Real totalPopulation "Total population: elite and commoners. No people";

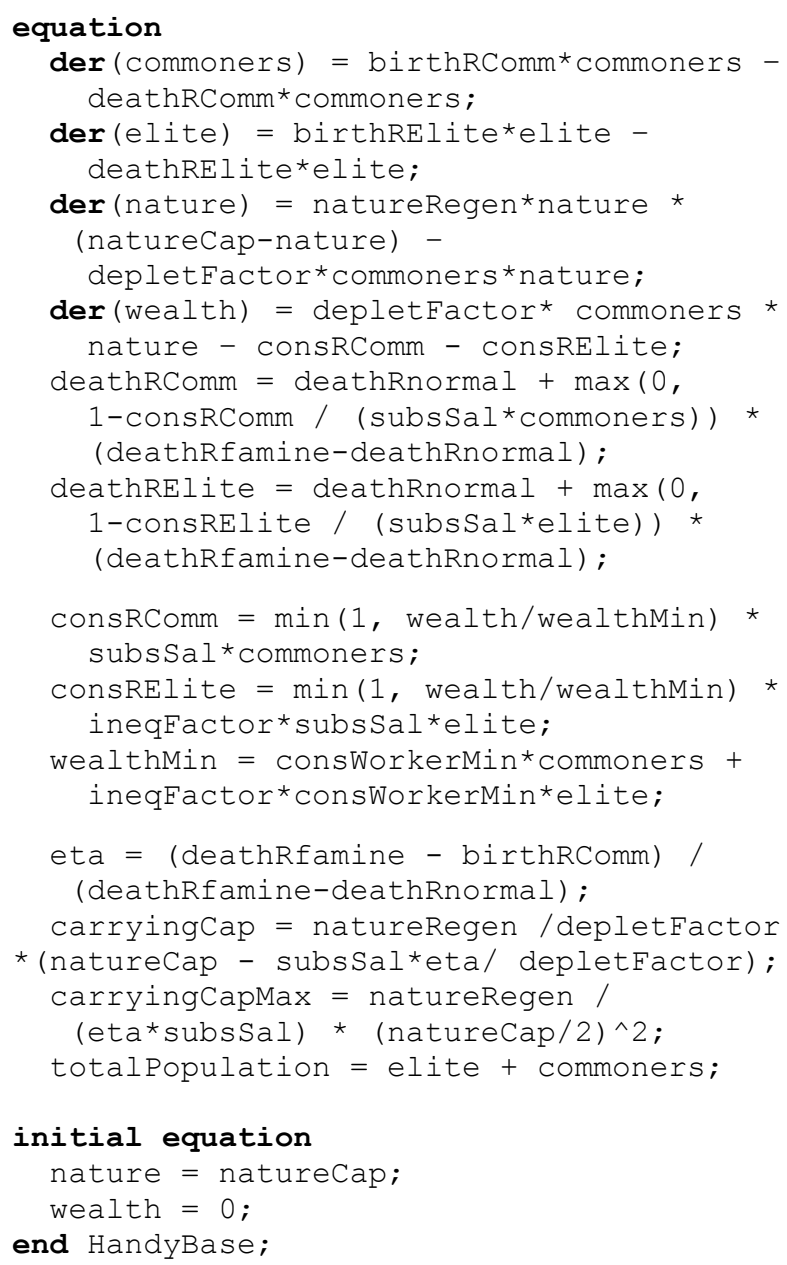

\subsection{Types of Societies, Simulation Methodology and Scenarios}

Handy has been applied to three types of societies:

- Egalitarian society - with no elites, i.e., the elite population elite $=0$. Scenario models HandyEgall to ModelEgal4.

- Equitable society - with workers (commoners) and elites (non-workers), where both groups earn the same per person, i.e., ineqFactor $=1$. Scenario models HandyEquit1 to HandyEquit5.

- Unequal society -with commoners and elites, where elites consume more per capita than commoners, i.e., ineqFactor $>1$. Scenario models HandyUnEq1 to HandyUnEq4.
Several scenarios will be studied for each kind of society. The default values for parameters and initial values for state variables used in these scenarios are those presented in Table 1 and Table 3.

The thought experiments are performed as simulations, the results of which are presented in Sections 4.3 and 4.5 , respectively. Within each type of society, different scenarios are studied by varying the rate of depletion per worker called depletFactor (short for depletion factor).

For each type of society, an equilibrium state can be reached, in which all derivatives go to zero, and consequently, all the system's variables settle into a steady state.

Under that condition, the carrying capacity can be maximized if nature's regeneration rate is maximal. According to the equation for nature, the latter is satisfied when nature=natureCap/2. The depletion factor depletFactor is at the optimal level depletFactorEq, when it produces a steady state with a maximum sustainable population.

The scenarios below explore the consequences of different types of societies as they behave optimally or sub-optimally, determined by the depletion factor.

\subsection{Simulation Scenarios of an Egalitarian So- ciety}

For this type of society, the optimal value for the depletion factor that maximizes the carrying capacity (carryingCap) is depletFactorEq=6.67E-6 (derived analytically). Below is the HandyEgalitarianBase model that is being inherited by all Egal models.

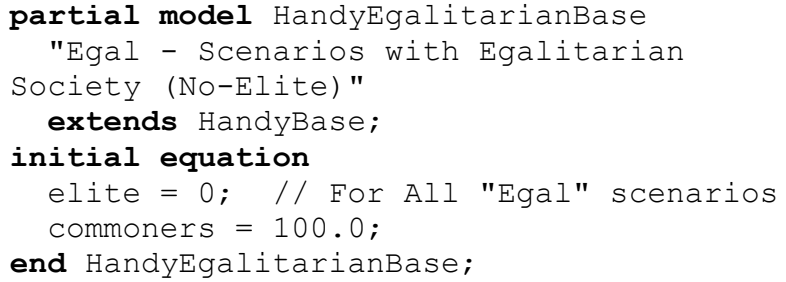

Scenario Model HandyEgal1: "Soft landing to Equilibrium"

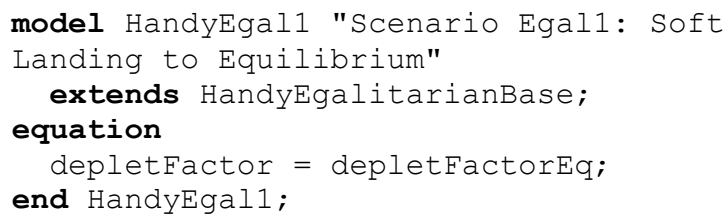

By making depletFactor=depletFactorEq the results in Figure 3 below are obtained:

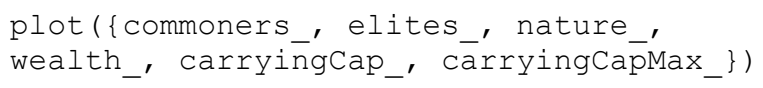




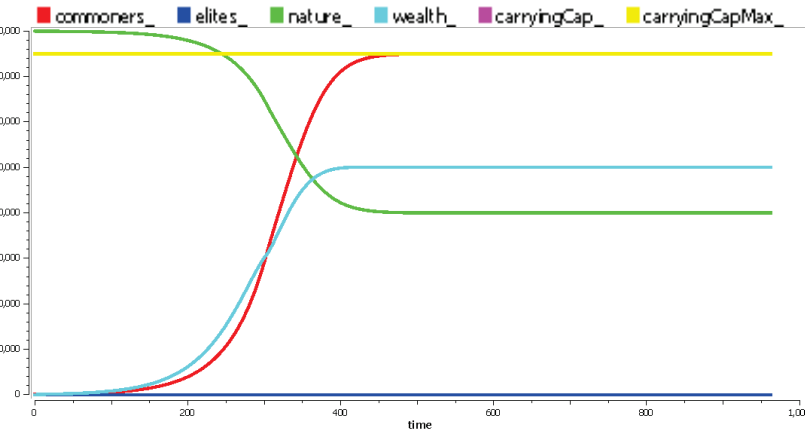

Figure 3. Soft landing to equilibrium, with optimal nature depletion factor. Simulation of HandyEgal1.

In this example, maximum nature regeneration can support a maximum sustainable depletion rate (pollution) and a steady maximum population (commoners) equal to the carrying capacity (carryingCap).

Scenario Model HandyEgal3: "Cycles of Prosperity and Collapse"

In this scenario we increase the depletion intensity by selecting depletFactor $=4$ * depletFactorEq.

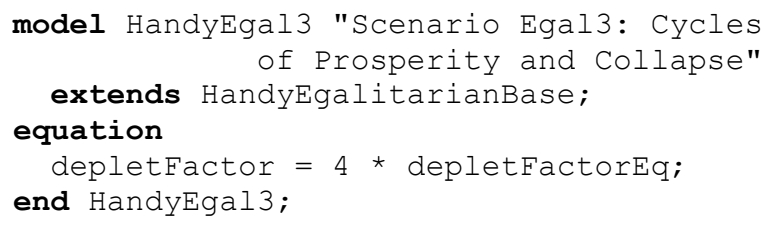

The results obtained are shown in Figure 4 below:

plot (\{commoners_, elites_, nature_, wealth_, carrying'cap_, carryingCap̄Max_\})

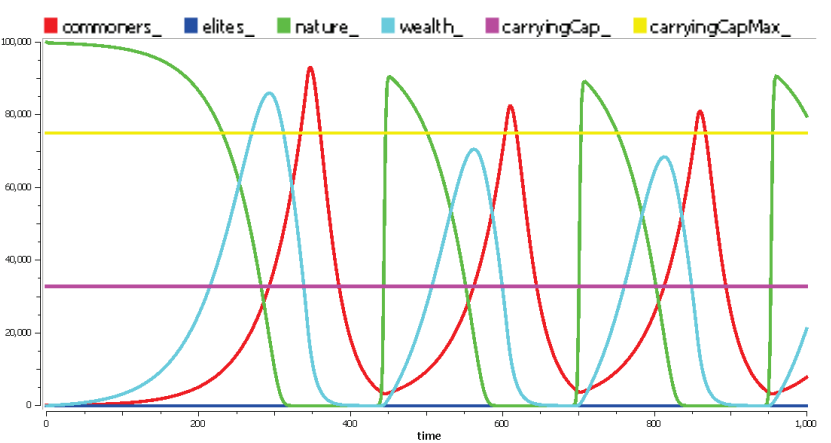

Figure 4. Cycles of Prosperity and Collapse. Simulation of HandyEgal3.

The oscillatory mode exhibits "reversible collapses". This scenario can be seen as belonging to a "limit cycle" for the values of the depletion factor. If depletFactor increases even further, the system changes into a different mode, one of "irreversible" collapse, with only one overshoot-and-collapse cycle. This is obtained in the scenario HandyEgal 4.

\section{Additional Scenarios in an Egalitarian Type of Society}

There are also scenarios models HandyEgal2 "Oscillatory Approach to Equilibrium" and HandyEgal 4 "Full
Collapse", corresponding to depletFactor = $2.5 *$ depletFactorEq and depletFactor $=$ $5.5 *$ depletFactorEq, respectively. The reader is encouraged to test these models.

\subsection{Simulation Scenarios of an Equitable Socie- ty-Workers and Non-Workers with Equal access to Wealth}

In this society, Non-Workers and Workers have equal access to wealth, i.e., ineqFactor $=1$. The nonworkers' privilege is that they get access to wealth without having to work (in that sense, they are regarded as the Elites). The initial population of non-workers is elite $=25$. As before, we look for an equilibrium situation where the depletion factor is set to its optimal value, which for this society is depletFactor $=$ depletFactorEq $=8.33 \mathrm{E}-6$. This produces the first scenario HandyEquit1 model shown further below, an adaptation of the HandyEquitableBase model which is inherited by all Equit scenario models.

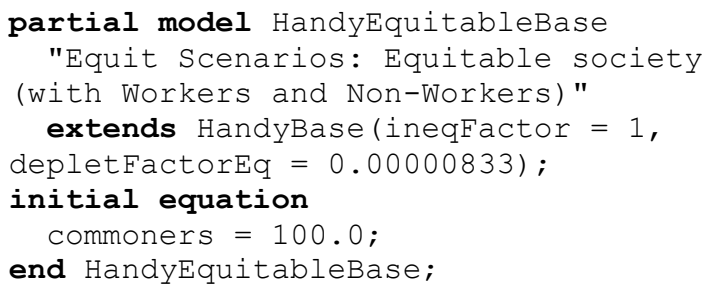

Scenario HandyEquit1: "Soft Landing to Optimal Equilibrium"

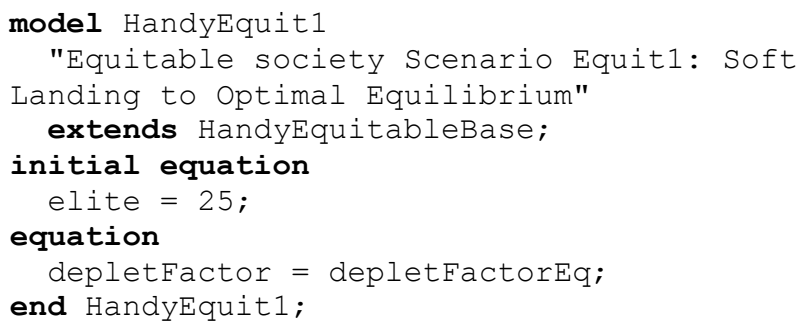

We simulate and plot: plot (\{commoners_, elites_, nature_, wealth_, carryingCap_, carryingCapMax_\})

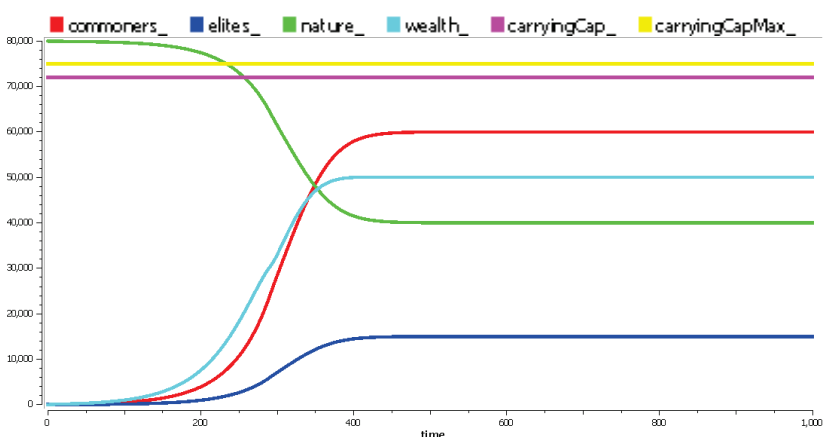

Figure 5. Equilibrium in the presence of workers (Commoners) and non-workers (Elites) with equal consumption per person. Simulation of HandyEquit 1 . 
In Figure 5, the sum of worker and non-worker populations reaches the maximum carrying capacity. The optimal depletion factor in this society is bigger than that of an egalitarian society analyzed in the previous section. This is due to an increased amount of production required from the workers to sustain the non-workers.

Scenario HandyEquit2: "Oscillatory Approach to Equilibrium"

If the depletion factor is further increased to depletFactor $=2.64 *$ depletFactorEq we obtain an oscillatory behavior. The results are shown in Figure 6

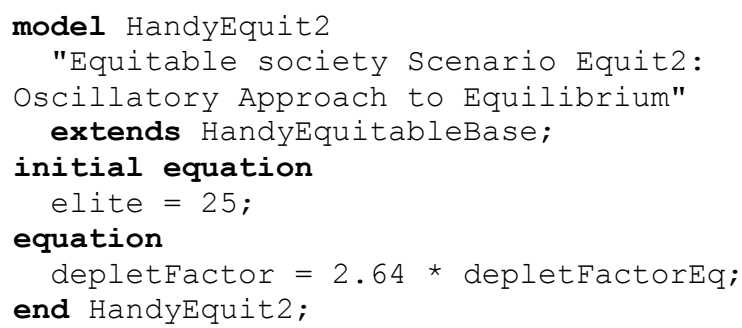

We simulate and plot:

plot (\{commoners,, elites_, nature,' wealth_, carryingCap_, carryingCap̄Max_\})

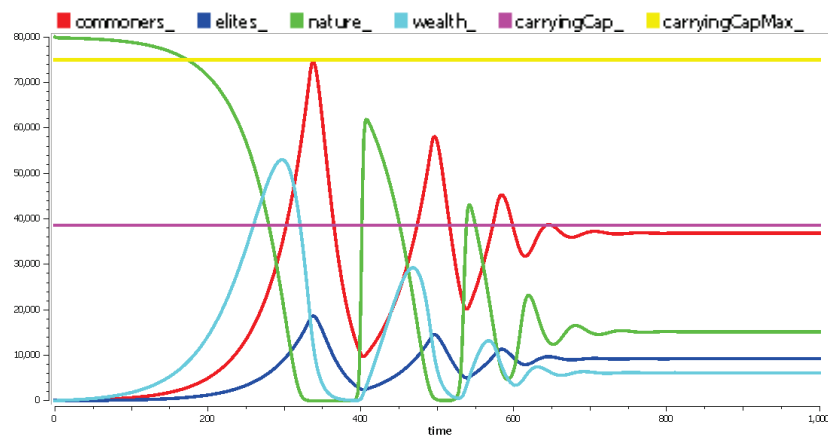

Figure 6. Oscillatory convergence to equilibrium with tolerable overshoot. Simulation of HandyEquit2.

Now, the carrying capacity (carryingCap) is lower than the maximum carrying capacity, but the total population approaches a steady state value after an oscillatory phase.

\section{Additional Scenarios in an Equitable Type of Society}

There are also scenarios HandyEquit3 "Cycles of Prosperity, Overshoot and Collapse", HandyEquit4 "Full Collapse", and HandyEquit5 "Preventing a Full Collapse by Decreasing Average Depletion per Capita".

The reader is encouraged to also try these models, which include experimenting with different values of the depletion factor, and in the case of HandyEquit5, increasing the ratio of non-workers to workers.

\subsection{Simulation Scenarios of an Unequal Socie- ty-Elites and Commoners with Different Access to Wealth}

The unequal society appears to be closer to the status of our current world. The inequality factor ineqFactor is made to vary from 10 to 100 in the unequal scenarios.

We will reproduce a pair of experiments intended to show the effects of changing birth rates and inequality as a means for moving from an unsustainable to a sustainable mode of behavior.

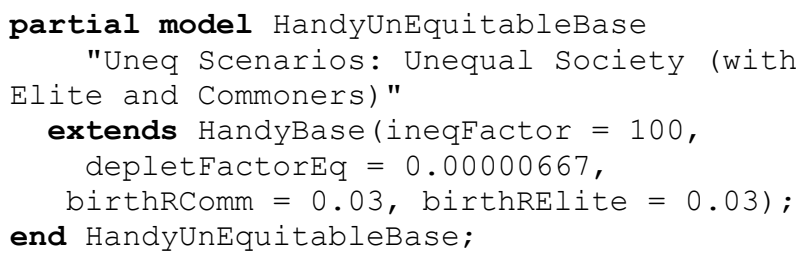

Scenario HandyUneq2: "Full Collapse"

In this scenario the inequality factor is set very high: ineqFactor $=100$. Also, the initial seed for the population of the Elite is set to elite $=0.2$ and a large depletion factor (including pollution effects) depletFactor $=1 \mathrm{E}-4$ is selected. The result is a full collapse with no recovery, as shown in Figure 7 below.
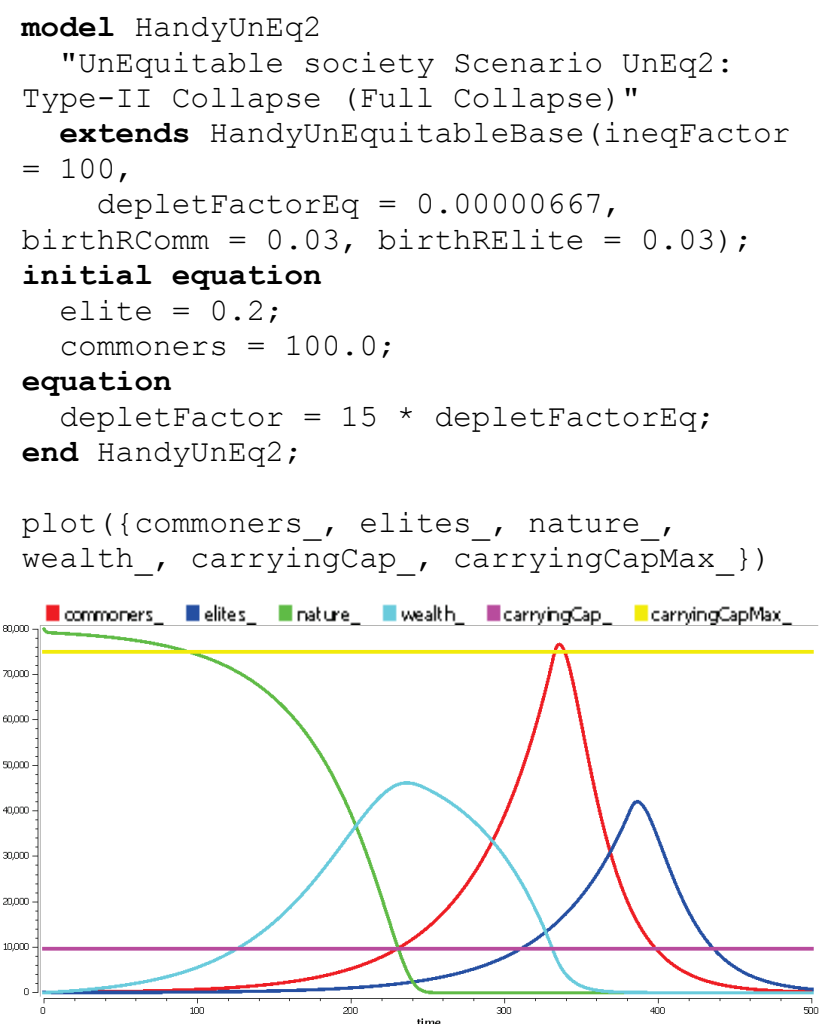

Figure 7. Full collapse due to over-depletion and high levels of inequality (ineqFactor=100). Simulation of HandyUnEq2. 
As soon as the Commoners' population surpasses the carrying capacity, wealth starts to decline and never again recovers. Before disappearing, Elites remain insensitive to the wealth's fast decrease for a long period after the Commoners' population starts its massive decline. This is possible due to the unequal access to wealth, which is a hundred times larger than that of the Commoners.

\section{Scenario HandyUneq3: "Soft Landing to Optimal Equilibrium"}

In this scenario several parameters and initial values are changed with respect to the previous case.

- Inequality is reduced by a factor of ten, yielding ineqFactor $=10$.

- The depletion factor is set to its equilibrium value, derived analytically: depletFactor = depletFactorEq $=6.35 \mathrm{E}-6$, much lower than in the previous scenario.

- The initial values for the population are set as commoners $=10000$ and elite $=3000$.

- The birth rates are assumed to be controllable and selected as birthRComm $=0.065$ and birthRElite $=0.02$.

The results are shown below:

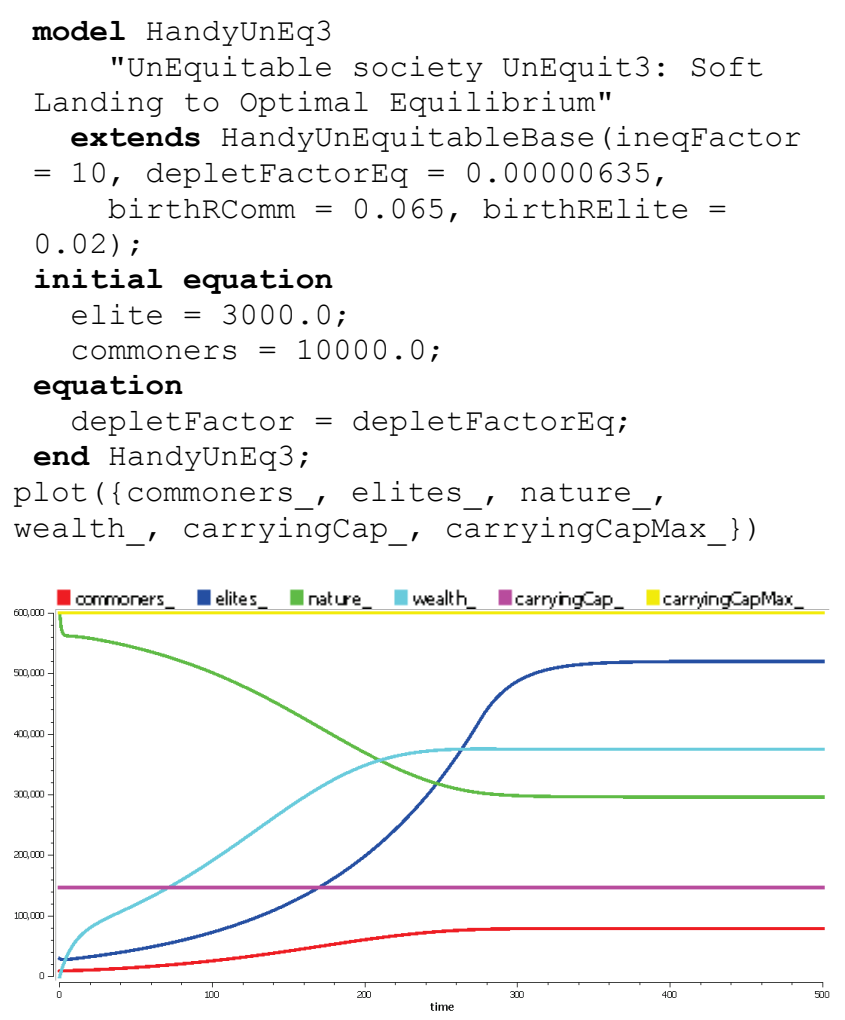

Figure 8. Unequal society reaching a sustainable equilibrium with moderate inequality (ineqFactor $=10$ ) and birth rate control. Simulation of HandyunEq3.
The new set of parameters produces a sustainable equilibrium in an unequal society. It represents an example of implementation of policies that simultaneously limit inequality and allow birth rates to remain below critical levels. In this model inequality and birth rate are separate but simultaneous measures. In fact, in the real world high birth rates in poor societies are often a consequence of inequality.

\section{Additional Scenarios for an Unequal Type of Society}

There are also scenarios HandyEquit1 "Collapse with Recovery of Nature" and HandyEquit4 "Oscillatory Approach to Equilibrium", which perform experiments with different values of the depletion factor, birth rates, and values of initial populations.

\section{A Short Look at the World3 Model}

World3 is a rather complex world model and therefore, its realm, scope, and objectives are quite different from those of Handy. The compiled version contains 41 state variables and 245 algebraic variables, with the same number of equations, representing many facets of human society and global ecological and economic dynamics.

The model is available as part of the SystemDynamics.WorldDynamics library [1], developed by François Cellier and his students. They made a documented Modelica version of World3 by translating the original model from its version in Stella.

The SystemDynamics.WorldDynamics.World3 model is represented as a Modelica package that implements Meadows et al World3 model. World3 was first described in the 1972 book Limits to Growth: A Report for the Club of Rome's Project on the Predicament of Mankind [5].The book has seen two later editions, one published in 1992 (20-year edition), and the most recent one published in 2004 (30-year edition) [7]. Each of these new editions were accompanied by an updated model. The model in this Modelica World3 version is the newest model discussed in the 2004 edition Limits to Growth: The 30-Year Update.

Whereas Jay Forrester listed his entire mathematical model in his book World Dynamics, Meadows et al in Limits to Growth only discussed the results obtained using their model. The mathematical model itself was not listed. The main reason was to make the book more accessible to a wider public. Another reason is that World3 is considerably more complex than World2, and consequently, a thorough discussion of all aspects of the model would have taken up much more space in the book. Instead, the authors published a separate book of 637 pages in 1974: Dynamics of Growth in a Finite World [6] that describes all facets of the model. 
Since the World3 model is rather complex, it was subdivided into 13 different sectors (i.e., submodels) describing aspects of the following: 1) arable land dynamics, 2) food production, 3) human ecological footprint, 4) human fertility, 5) human welfare index, 6) industrial investment, 7) labor utilization, 8) land fertility, 9) life expectancy, 10) non-recoverable resource utilization, 11) pollution dynamics, 12) population dynamics, and 13) service-sector investment.

In the overall main model, one submodel of each class is placed on the screen, and the individual submodels are connected appropriately (Figure 9).

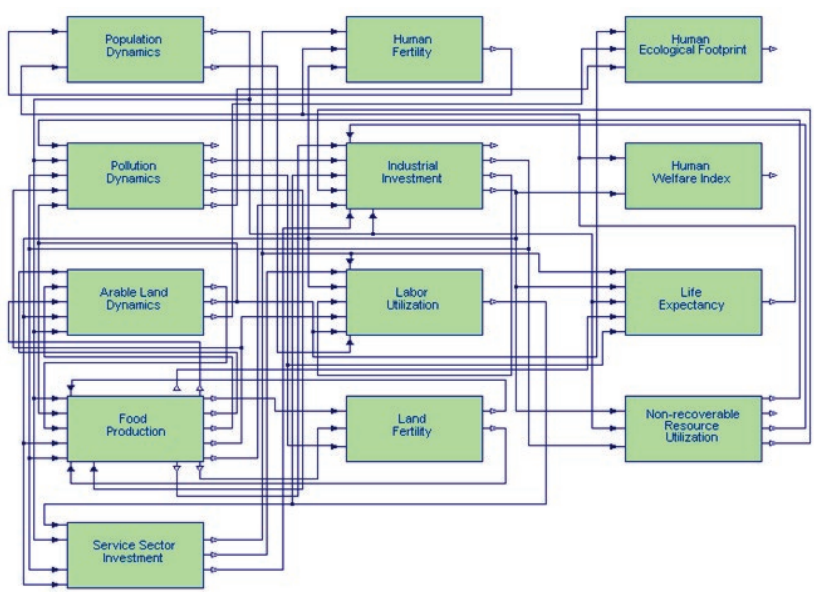

Figure 9. Overall World 3 model.

The submodel World3. Land_Fertility, for example, is depicted below in Figure 10.

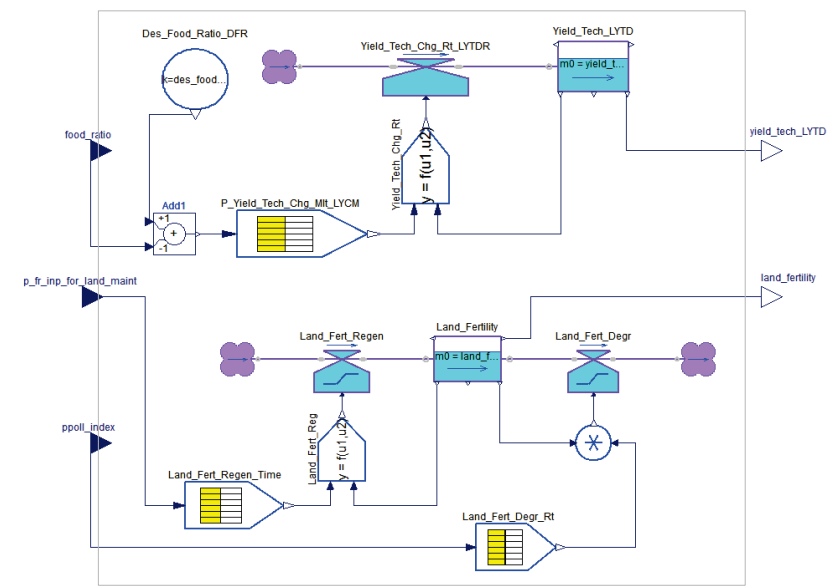

Figure 10. A submodel of World3:

SystemDynamics.World3. Land_Fertility.

This submodel has 3 Inputs and 2 outputs. Two state variables, Land_Fertility and Yield_Tech, are represented by means of "Level" blocks, which have the ability to accumulate. Levels play the role of the state variables in a differential equation. Levels are in turn driven by the input and/or output "Rates" connected to the left and right sides, respectively, determining the velocities at which the accumulated quantity increases or decreases. These Rates, in turn can be functions of other variables, including the state of the Level itself. Said functions are defined and interconnected following a block diagram approach, resorting to a vast library of pre implemented blocks such as Non-Linear Functions, Table Lookup Functions, Gains, Multipliers, etc.

The "Clouds" connected to the rates have only a pictorial purpose, expressing the fact that a source and/or a sink always exists with the physical ability to provide and/or absorb material without limits. The latter can be considered the conceptual "boundaries" of the model, as no detail is given for the processes taking place in material supply or consumption at these ends.

Whereas the World2 model lumps the entire population into a single state variable, the World 3 model offers a demographic population dynamics model that distinguishes between children and adolescents, young adults of child-bearing age, older adults who are still integrated into the work force, and the retired population.

The capital investment is subdivided into investments in the military/industrial complex, in the service sector, and in agriculture.

Both the natural resources and pollution models have been upgraded by including changes in technology as factors influencing the depletion of resources and the release of pollutants. This is meaningful as improved technology may enable us to use the available resources more efficiently, and may also make it possible to produce goods in a cleaner fashion.

\section{Scenarios with World3}

The book Limits to Growth: The 30-Year Update, describes eleven scenarios based on different sets of assumptions. These scenarios are also part of the Modelica World3 model. We shall briefly discuss three of these scenarios:

- Scenariol is the so-called "Standard Run". This is the original World3 model with basic assumptions and without any adjustments.

- Scenario2 is the same as Scenariol, but with twice as much non-recoverable resources initially available, i.e., more oil, coal, metal, etc.

- Scenariog is based on Scenario2 but applies a number of measures to avoid collapse and to try to transform the human society into a sustainable one.

The three scenarios are simulated between year 1900 and 2200 .

Unfortunately, both Scenario1 and Scenario2 lead to population collapse rather quickly, as depicted in Figure 11. Doubling the non-renewable resources in Sce- 
nario2 does not help at all (it delays the collapse, but makes it steeper). The population increases a little further, but the subsequent collapse is even more severe, primarily due to increased levels of pollution. The current developments in our world unfortunately seem to be rather close to Scenario2.

If a collapse indeed should occur, will it be as quick as depicted in Figure 11? That must not necessarily be the case. The World3 model assumptions may not be completely valid during such a stressful transition period. Political decisions, rationing policies, etc., may lead to decreasing resource consumption and create a slower, more controlled contraction to a sustainable level of consumption.

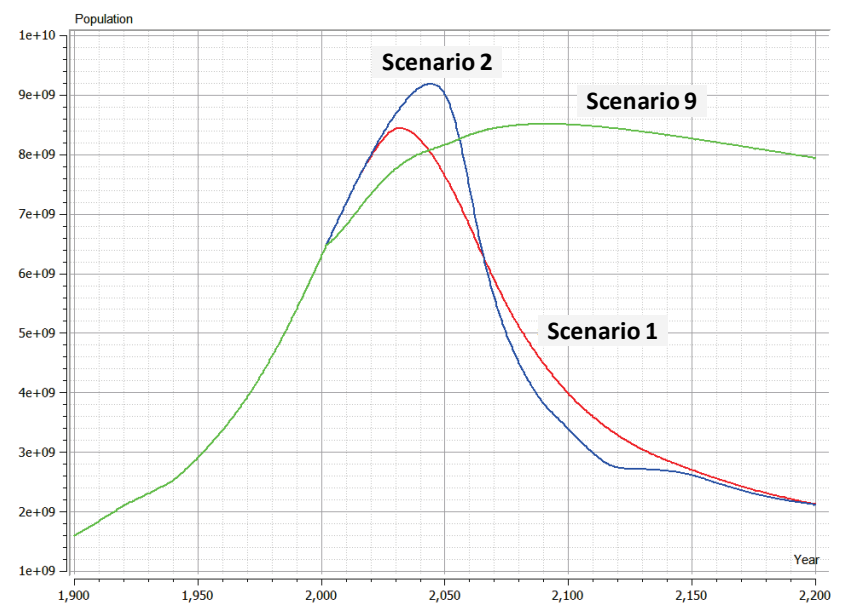

Figure 11. The world population according to Scenarios 1, 2, and 9 in the World 3 model.

How can a collapse be avoided? Scenario9 applies the following aggressive technology development plan:

- Increased industrial resource efficiency, i.e., a reduction in the use of non-renewable material and energy, a $4 \%$ resource decrease/year.

- Arable land protection, e.g., to decrease and prevent land erosion, and to preserve existing land.

- Agricultural yield enhancement, a 4\% increase/year.

- Pollution reduction, a 4\% decrease/year.

Additionally, a program for birth control is implemented with an average number of two children per family. The scenario furthermore assumes that there is a certain capital cost to implement these technologies, and that 20 years are required for their full implementation.

The scenariog simulation to year 2200 shows a sustainable society. However, if the simulation continues to the year 2500 , we observe that it is hard to maintain sustainability with a large human population combined with high standards of living. If the size of the population is gradually decreased back to its level of about a hundred years ago, the chances for being able to maintain high standards of living are much better.
Another issue is model validity. A number of assumptions behind the World3 model may not be valid that far into the future. However, one fact is certain: the human race must, in the long run, live sustainably on planet Earth due to limitations in resources, energy, and space. Consumption overshoots like the current one can only be temporary.

\section{Conclusion}

The Handy model has the remarkable feature of providing a minimal structure helpful for intuitive understanding of the human-nature interaction. It can produce a rich variety of dynamical modes, leading to non-trivial scenarios. This combination is seldom found in most world models, where the quest for including more details comes at the price of lack of intuitiveness.

Handy is very useful as a "conceptual" model that can trigger varied interpretations and discussions comparing qualitatively different scenarios.

However, the goal of Handy is not to make quantitative short-term forecasts of the world state, but to assess qualitatively the impacts of various factors such as inequality and depletion on the long-term behavior of human societies.

The explicit stratification of social classes guides the interpretations to an essential playground needed to discuss the fate of human population.

Also worth mentioning is the fact that human societies developed a degree of cultural and technological complexity that prevents treating them in the same way as an animal species.

Accumulation of wealth (human's ability to save throughout generations) is a major distinction which allows for irreversible population collapses, whereas with predation only, the predator-prey type of models can only produce cyclic oscillations (i.e., partial collapses).

Several senior modelers have remarked that it is not reasonable to expect that any world model will correctly predict how the world will develop after the onset of collapse, as unpredictable social disruptions and/or restructurings would result that invariably invalidate most of the model's assumptions, parameters and even structures that might have described pre-collapse phases in a credible way. Such social reshaping will have its dynamics determined by interactions between different social classes, and in that regard, again, the Handy approach of disaggregating them in at least two strata represents a valuable contribution to future world modeling.

Moreover, we want to draw the reader's attention to the aggregation made in Handy of natural renewable and non-renewable resources into a stock variable. This 
paves the way for attaining scenarios that look sustainable in the very long run (i.e., when simulating "to infinity"). Based on the principles of thermodynamics it can be shown that in the long run the maximum carrying capacity of humans on earth will be determined only by the remaining renewable resources (once we have depleted all the non-renewables for good).

If we consider renewable and non-renewable resources independently of each other (along with the commoners, the elites, and their inequality factor), we may be able to use the resulting world model to investigate scenarios that offer possible answers to an additional highly relevant question. How should we best use the non-renewable resources to attain a society with minimum inequalities, for when the time comes that humanity must rely on renewable resources only? To investigate such a goal, the inequality factor should be altered from a constant parameter to a variable. Although models presented in this paper are from different classes (minimal Handy vs. more complex, realistic world model, World3), their conclusions are similar. In the long run, not so far into the future, humanity must change to living sustainably on planet Earth. This change can occur either as a planned gradual transition, preserving well-functioning societies, or as a more disruptive, unplanned transition resulting in a less pleasant society with a reduced ecological capacity.

\section{Acknowledgments}

This work has been supported by the Swedish Governmental Agency for Innovation Systems (Vinnova) within the ITEA2 MODRIO project, and by the Swedish Research Council (VR).

\section{References}

[1] Cellier, François. World3 in Modelica: Creating System Dynamics Models in the Modelica
Framework. In Proceedings of the 6th International Modelica Conference, Bielefeld, Germany. 2008.

[2] Fritzson, Peter. Principles of Object Oriented Modeling and Simulation with Modelica 2.1, 940 pages, ISBN 0-471-471631, Wiley-IEEE Press. Feb. 2004.

[3] Lotka, Alfred J. Elements of Mathematical Biology. Dover Publications, New York, 1956.

[4] Malthus, T. An Essay on the Principle of Population. Printed for J. Johnson, in St. Paul's ChurchYard, London, 1798. (Reprint. Amherst, NY: Prometheus Books, 1998).

[5] Meadows D., Meadows D., Randers J., and Behrens III W.W. Limits to Growth: A Report for the Club of Rome's Project on the Predicament of Mankind. 205p. Universe Books, New York, 1972.

[6] Meadows D., Behrens III W.W., Meadows D., Naill R.F., Randers J., and Zahn E.K.O. Dynamics of Growth in a Finite World. 637p. WrightAllen Press, 1974.

[7] Meadows D., Randers J., and Meadows D. Limits to Growth: The 30-Year Update. 368p. Chelsea Green, 2004.

[8] Modelica Association. Modelica—A Unified Object-Oriented Language for Physical Systems Modeling: Language Specification Version 3.2 rev 2. Available at http://www.modelica.org, August, 2013.

[9] Motesharrei S., Rivas J., and Kalnay E. A Minimal Model for Human and Nature Interaction. Accepted for publication (expected 2014) Journal Ecological Economics. 